\title{
Symmetry breaking of an elliptic equation in expanding annuli
}

\author{
Zongming Guo, Linfeng Meiø and Fangshu Wan
}

\begin{abstract}
We obtain non-radial bifurcation from radial solutions of a semilinear elliptic equation in expanding annuli of $\mathbb{R}^{N}$. To obtain the main results, we use a blow-up argument via the Morse index of the regular entire solutions of the equation

$$
-\Delta u=\lambda u^{p} \quad \text { in } \quad \mathbb{R}^{N} .
$$

The main results of this paper can be seen as applications of the properties of regular entire solutions of (0.1).

Mathematics Subject Classification. Primary 35K20, 35R35; Secondary 35J60.
\end{abstract}

Keywords. Expanding annuli, Bifurcation, Symmetry breaking, Morse index, Supercritical exponent.

\section{Introduction}

We study symmetry breaking of radial solutions to the equation

$$
\begin{cases}-\Delta u=\lambda f(u) & \text { in } D_{R}, \\ u>0 & \text { in } D_{R}, \\ u=0 & \text { on } \partial D_{R},\end{cases}
$$

where $D_{R}=\left\{x \in \mathbb{R}^{N}: R<|x|<R+1\right\}$ with $N \geq 2$,

$$
R \geq\left[(N-1)^{\frac{1}{N-2}}-1\right]^{-1} \quad \text { for } \quad N \geq 3 ; \quad R \geq[e-1]^{-1} \quad \text { for } \quad N=2,
$$

$\lambda$ is a positive parameter, $f$ satisfies the conditions:

(A-1) $f \in C^{2}\left(\mathbb{R}^{1}\right)$ and $f(u)>0$ for $u \geq 0$,

$(\mathrm{A}-2) f^{\prime}(u)>0$ and $f^{\prime \prime}(u)>0$ for all $u \geq 0$,

\footnotetext{
* Research of the first author is supported by NSFC (11171092, 11571093); Research of the second author is supported by NSFC (11371117).
} 
(A-3) $\lim _{u \rightarrow \infty} \frac{f(u)}{u^{p}}=1$ with $p \in\left(1, p_{c}(N)\right) \backslash\left\{\frac{N+2}{N-2}\right\}$, where

$$
p_{c}(N):= \begin{cases}\infty & \text { if } 2 \leq N \leq 10, \\ \frac{(N-2)^{2}-4 N+8 \sqrt{N-1}}{(N-2)(N-10)} & \text { if } N \geq 11\end{cases}
$$

Note that

$$
1<\frac{N+2}{N-2}<p_{c}(N) \quad \text { for } \quad N \geq 3
$$

and $p_{c}(N)$ is a root of the equation

$$
\frac{2 p}{p-1}\left[N-2-\frac{2}{p-1}\right]=\frac{(N-2)^{2}}{4} .
$$

A typical example for $f$ satisfying (A-1)-(A-3) is $f(u)=|u+1|^{p-1}(u+1)$.

We will obtain bifurcations of nonradial solutions from the radial solutions of (1.1). It is well-known that if $u \in C^{2}\left(\mathbb{R}^{N}\right)$ is a nonnegative solution of the equation

$$
-\Delta u=\lambda u^{p} \quad \text { in } \quad \mathbb{R}^{N},
$$

then $u \equiv 0$ in $\mathbb{R}^{N}$ provided that $1<p<\frac{N+2}{N-2}$; if $u \in C^{2}\left(\mathbb{R}^{N}\right)$ is a stable nonnegative solution of (1.3), then $u \equiv 0$ in $\mathbb{R}^{N}$ provided that $p=\frac{N+2}{N-2}$ (see $[10,12]$ ) (note that $(1.3)$ admits solutions with finite Morse index provided $p=\frac{N+2}{N-2}$ (see [10])); and if $u \in C^{2}\left(\mathbb{R}^{N}\right)$ is a positive solution of (1.3), then $i(\lambda, u)=\infty$ provided that $\frac{N+2}{N-2}<p<p_{c}(N)$ (see $\left.[8,9,11,12]\right)$, where $i(\lambda, u)$ is the Morse index at $(\lambda, u)$. (Note that under a scaling $v=\lambda^{1 /(p-1)} u$, then $-\Delta v=v^{p}$.)

Let $u$ be a solution to the problem

$$
\begin{cases}-\Delta u=\lambda f(u) & \text { in } \quad \Omega \\ u=0 & \text { on } \quad \partial \Omega\end{cases}
$$

where $\Omega \subset \mathbb{R}^{N}$ is a bounded or unbounded domain, $\lambda>0$. Denote by $Q_{u}$ the bilinear form associated to $u$, i.e.

$$
Q_{u}(\phi, \psi)=\int_{\Omega} \nabla \phi \cdot \nabla \psi d x-\int_{\Omega} \lambda f^{\prime}(u) \phi \psi d x, \quad \phi, \psi \in H_{0}^{1}(\Omega) .
$$

The Morse index at $(\lambda, u)$, denoted by $i(\lambda, u)$, is the maximal dimension of a subspace $X \subset C_{0}^{1}(\Omega)$ such that

$$
Q_{u}(\psi, \psi)<0 \quad \forall \psi \in X \backslash\{0\} .
$$

This is equivalent to say that $i(\lambda, u)$ is the number of negative eigenvalues of $-\Delta-\lambda f^{\prime}(u) I$ computed with their multiplicity. If $\Omega=\{x: a<|x|<b\}$ with $b>a>0$ and $u$ is a radial solution of (1.4), the radial Morse index at $(\lambda, u): i_{\text {rad }}(\lambda, u)$ is the number of negative eigenvalues of the problem

$$
\left\{\begin{array}{l}
\psi^{\prime \prime}+\frac{N-1}{r} \psi^{\prime}+\lambda f^{\prime}(u) \psi=-\mu \psi, \quad r \in(a, b), \\
\psi(a)=\psi(b)=0
\end{array}\right.
$$

Problem (1.1) arises from many branches of mathematics and applied mathematics. It was studied by many authors, for example, $[1-3,5,6,13-19$, $21,22]$ and the references therein. 
It is known from Theorem 2.11 of [16] that, for any $R$ satisfying (1.2), there exists $\lambda_{R}^{*}>0$ such that (1.1) has exactly two positive radial solutions $0<\underline{u}_{R}^{\lambda}<\bar{u}_{R}^{\lambda}$ for $\lambda \in\left(0, \lambda_{R}^{*}\right)$, exactly one $u_{R}^{*}$ at $\lambda=\lambda_{R}^{*}$, and none for $\lambda>\lambda_{R}^{*}$. It is well-known that $\underline{u}_{R}^{\lambda}\left(0<\lambda<\lambda_{R}^{*}\right)$ and $u_{R}^{*}$ are stable. Moreover, $u_{R}^{*}$ is the unique solution of (1.1) at which the first eigenvalue of the linearized problem is 0 . Note that $\bar{u}_{R}^{\lambda}$ is radially nondegenerate for $\lambda \in\left(0, \lambda_{R}^{*}\right)$. To see this, we notice that if $\mu$ is an eigenvalue of the problem

$$
\left\{\begin{array}{l}
-\phi^{\prime \prime}(r)-\frac{N-1}{r} \phi^{\prime}(r)-\lambda f^{\prime}\left(\bar{u}_{R}^{\lambda}\right) \phi=\mu \phi, R<r<R+1, \\
\phi(R)=\phi(R+1)=0,
\end{array}\right.
$$

then $\mu$ is simple. By the bifurcation theory on the critical point of odd multiplicity, $[24,25]$, if there is some $\mu=0$, it is actually a secondary bifurcation point, i.e. there is a new radial solution branch of (1.1) coming from $\left(\lambda, \bar{u}_{R}^{\lambda}\right)$ (note that the nonlinearity of (1.1) is convex). This contradicts the fact that (1.1) has exactly two radial solutions for $\lambda \in\left(0, \lambda_{R}^{*}\right)$. Meanwhile, it is known from [4] that $i\left(\lambda, \underline{u}_{R}^{\lambda}\right)=i_{\text {rad }}\left(\lambda, \underline{u}_{R}^{\lambda}\right)=0$ for $\lambda \in\left(0, \lambda_{R}^{*}\right], i\left(\lambda, \bar{u}_{R}^{\lambda}\right) \geq 1$ and $i_{\text {rad }}\left(\lambda, \bar{u}_{R}^{\lambda}\right)=1$ for $\lambda \in\left(0, \lambda_{R}^{*}\right)$.

Arguments similar to those in the proof of Theorem 1.1 of [16] imply that the similar result is also true for the problem

$$
\left\{\begin{array}{l}
-u^{\prime \prime}=\lambda f(u), \quad \text { in } \quad(0,1), \\
u(0)=0=u(1),
\end{array}\right.
$$

i.e. there exists a $\lambda_{0}^{*} \in(0, \infty)$ such that problem (1.5) admits no solutions for $\lambda>\lambda_{0}^{*}$, one and only one solution $u_{0}^{*}$ for $\lambda=\lambda_{0}^{*}$ and exactly two solutions $0<\underline{u}_{0}^{\lambda}<\bar{u}_{0}^{\lambda}$ for $0<\lambda<\lambda_{0}^{*}$. It is well-known that $\underline{u}_{0}^{\lambda}\left(0<\lambda<\lambda_{0}^{*}\right)$ and $u_{0}^{*}$ are stable and the Morse index of $\bar{u}_{0}^{\lambda}\left(0<\lambda<\lambda_{0}^{*}\right)$ is not less than 1. Moreover, $u_{0}^{*}$ is the unique solution of (1.5) at which the first eigenvalue of the linearized problem is 0 . (Note that when $N=1$, i.e. equation (1.5), $\rho(s)$ and $s_{0}$ as defined in [16] are $\rho(s) \equiv 1$ and $s_{0}=0$. We easily see that (2.26) in [16] holds).

In this paper, we are interested in studying the asymptotic behaviors of the radial solutions and bifurcation of nonradial solutions from a radial solution of (1.1) when $R$ varies. To do these, we first need to know the asymptotic behavior of $\lambda_{R}^{*}$ as $R \rightarrow \infty$. The main results of this paper are the following theorems.

Theorem 1.1. Let $f$ satisfy $(A-1)-(A-3)$. Then

$$
\lim _{R \rightarrow+\infty} \lambda_{R}^{*}=\lambda_{0}^{*}
$$

Moreover, for $\lambda \in\left(0, \lambda_{0}^{*}\right), \underline{u}_{R}^{\lambda}(R+t) \rightarrow \underline{u}_{0}^{\lambda}(t)$ uniformly for $t \in[0,1]$ as $R \rightarrow \infty$ and $u_{R}^{*}(R+t) \rightarrow u_{0}^{*}(t)$ uniformly for $t \in[0,1]$ as $R \rightarrow \infty$.

Theorem 1.2. Let $f$ satisfy $(A-1)-(A-3)$. Then for any fixed $\lambda \in\left(0, \lambda_{0}^{*}\right)$, there exist $R_{*}>1$ and a sequence $\left\{R_{j}\right\}$ with $R_{j} \geq R_{*}$ and $R_{j} \rightarrow \infty$ as $j \rightarrow \infty$ such that the Morse index $i\left(\lambda, \bar{u}_{R_{j}}^{\lambda}\right) \rightarrow \infty$ as $j \rightarrow \infty$.

Theorem 1.3. Let $f$ satisfy $(A-1)-(A-3)$. Then for any fixed $\lambda \in\left(0, \lambda_{0}^{*}\right)$, there exists a sequence $\left\{R_{k}\right\}$ with $R_{k} \geq R_{*}$ and $R_{k} \rightarrow \infty$ as $k \rightarrow \infty$ such that $a$ nonradial bifurcation occurs at $\left(\lambda, R_{k}, \bar{u}_{R_{k}}^{\lambda}\right)$. 
Bifurcation and symmetry breaking of positive radial solutions of the equations with "positive exponent" in annular domains have been studied by many authors, see, for example, $[1,14-18]$ and the references therein. Lin ([16]) obtained non-radial bifurcation from radial solutions of the problem as (1.1) on a fixed annular domain. He also obtained symmetry breaking type results for a problem as (1.1) in varying annuli but the growth rate of $f(u)$ is subcritical or supercritical in a special range (see [17]). Bartsch et al. [1] obtained symmetry breaking type results for the Dirichlet problem of $-\Delta u=u^{p}$ in varying annuli without any extra restriction on $p>1$, but they used the fact that the radial solution of the problem is unique. Gladiali et al. [14] studied the Dirichlet problem of $-\Delta u=u^{p}+\lambda u$ on expanding annuli with any $p>1$, they also used the fact that the radial solution is unique. We will obtain symmetry breaking type results for (1.1) in varying annuli with a better assumption on the growth rate of the nonlinearity $f(u)$, but our problem admits multiple radial solutions for each fixed $\lambda>0$. It is easily seen that our assumption on the growth rate of the nonlinearity $f(u)$ is "sharp" in low dimensional case $(2 \leq N \leq 10)$. We think that our assumption on the growth rate of the nonlinearity $f(u)$ to obtain the symmetry breaking type results is also "sharp" even for higher dimensional case $(N \geq 11)$, but we can not give the proof here. To obtain our main results, we use a blow-up argument via the Morse index of the regular entire solutions of the Eq. (1.3).

\section{Preliminaries}

In this section we obtain some results on the spectrum of the linearized operator associated to a radial solution $u$ of the problem

$$
\begin{cases}-\Delta u=\lambda f(u) & \text { in } D, \\ u=0 & \text { on } \partial D,\end{cases}
$$

where $D=\left\{x \in \mathbb{R}^{N}: \alpha<|x|<\beta\right\}(0<\alpha<\beta<\infty), \lambda>0$. Note that $u$ depends on $\lambda$. The linearized operator at $u$ is defined as

$$
L_{u}=-\Delta-\lambda f^{\prime}(u) I .
$$

We recall that a solution $u$ to $(2.1)$ is degenerate if the equation

$$
\begin{cases}-\Delta z-\lambda f^{\prime}(u) z=0 & \text { in } D \\ z=0 & \text { on } \partial D\end{cases}
$$

admits nontrivial solutions.

In order to study the spectrum of the operator $L_{u}$, as in [1], we introduce the operators $\tilde{L}: H^{2}(D) \cap H_{0}^{1}(D) \rightarrow L^{2}(D)$,

$$
\tilde{L}_{u}:=|x|^{2}\left(-\Delta-\lambda f^{\prime}(u) I\right), \quad x \in D
$$

and $\hat{L}: H^{2}(\alpha, \beta) \cap H_{0}^{1}(\alpha, \beta) \rightarrow L^{2}(\alpha, \beta)$,

$$
\hat{L}_{u}(w):=r^{2}\left(-w^{\prime \prime}-\frac{N-1}{r} w^{\prime}-\lambda f^{\prime}(u) w\right) \quad r \in(\alpha, \beta) .
$$


Note that the eigenvalues of $\tilde{L}_{u}$ are given by

$$
\tilde{\mu}_{i}=\inf _{W \subset H_{0}^{1}(D), \operatorname{dim} W=i} \max _{w \in W, w \neq 0} \frac{\int_{D}|\nabla w|^{2}-\int_{D} \lambda f^{\prime}(u) w^{2}}{\int_{D}|x|^{-2} w^{2}}
$$

and the eigenvalues $\hat{\mu}_{i}$ of $\hat{L}_{u}$ are obtained in the same way just replacing the space $H_{0}^{1}(D)$ by $H_{0}^{1}(\alpha, \beta)$.

The proofs of the following lemmas can be obtained by arguments similar to those in the proofs of Lemmas 2.1, 2.2 and 2.3 in [14].

Lemma 2.1. Let $u$ be a radial solution to (2.1). Then the Morse index $i(\lambda, u)$ of $u$ is equal to the number of negative eigenvalues $\tilde{\mu}_{u}$ of the operator $\tilde{L}_{u}$.

Denoting by $\sigma(\cdot)$ the spectrum of a linear operator, we have:

Lemma 2.2. Let $u$ be a radial solution to (2.1), then

$$
\sigma\left(\tilde{L}_{u}\right)=\sigma\left(\hat{L}_{u}\right)+\sigma\left(-\Delta_{S^{N-1}}\right) .
$$

Let us denote by $\lambda_{k}, k=0,1,2, \ldots$ the eigenvalues of the operator $-\Delta_{S^{N-1}}$ and by $\phi_{k}$ the corresponding eigenfunctions. It is known that $\lambda_{k}=$ $k(k+N-2)$. Let us denote by $\hat{\mu}_{i}$ the eigenvalues of $\hat{L}_{u}$ and by $w_{i}$ the corresponding eigenfunctions with $\left\|w_{i}\right\|_{\infty}=1$.

Lemma 2.3. Let $u$ be a radial solution to (2.1) which is nondegenerate in the space $H_{0, \text { rad }}^{1}(D)$. Then problem $(2.2)$ has a nontrivial solution if and only if there exists $k \geq 1$ such that

$$
\hat{\mu}_{1}+\lambda_{k}=0 \text {. }
$$

Moreover the solutions $z$ to $(2.2)$ can be written as

$$
z(x)=w_{1}(|x|) \phi_{k}\left(\frac{x}{|x|}\right)
$$

where $w_{1}(r)$ is the first positive eigenfunction of $\hat{L}_{u}$ and $\phi_{k}(\theta)$ is the eigenfunction of $-\Delta_{S^{N-1}}$ relative to the eigenvalue $\lambda_{k}$.

\section{Asymptotic behaviors and Morse index of the radial solutions}

In this section, we consider the asymptotic behaviors and Morse index of the radial solutions of (1.1). Note that the domains $D_{R}$ are expanding annuli when $R$ varies. We will present the proof of Theorems 1.1 and 1.2 in this section.

Lemma 3.1. Let $0<a \ll 1$ be sufficiently small and $A_{a}=\{x: 1<|x|<$ $1+a\} \subset \mathbb{R}^{N}$. Then there is $0<\lambda_{a}^{*}<\infty$ such that for $\lambda \in\left(0, \lambda_{a}^{*}\right)$, the problem

$$
\begin{cases}-\Delta u=\lambda f(u) & \text { in } A_{a}, \\ u=0 & \text { on } \partial A_{a}\end{cases}
$$

admits exactly two radial solutions $0<\underline{u}_{\lambda}^{a}<\bar{u}_{\lambda}^{a}$; (3.1) admits exactly one radial solution $u_{*}^{a}$ for $\lambda=\lambda_{a}^{*}$; (3.1) does not admit a radial solution for $\lambda>\lambda_{a}^{*}$. Moreover, $\lambda_{a}^{*}$ is a decreasing function with respect to a and $\lim _{a \rightarrow 0^{+}} \lambda_{a}^{*}=\infty$. There exists $C>0$ such that

$$
\lambda_{a}^{*} \geq C a^{-2}
$$


Proof. We only need to show that $\lambda_{a}^{*}$ is a decreasing function with respect to $a$ and (3.2), the other claims are standard and known from Theorem 2.11 of $[16]$.

For any $a_{1}, a_{2}>0, a_{2}>a_{1}$, we see that $A_{a_{1}} \subset A_{a_{2}}$ and $u_{*}^{a_{2}}$ is a supersolution to the problem

$$
\left\{\begin{array}{lll}
-\Delta u=\lambda_{a_{2}}^{*} f(u) & \text { in } & A_{a_{1}} \\
u=0 & \text { on } & \partial A_{a_{1}}
\end{array}\right.
$$

This implies that (3.1) with $a=a_{1}$ admits a minimal radial solution for $\lambda=\lambda_{a_{2}}^{*}$. Note that $u \equiv 0$ is a subsolution to (3.3) (note $f(0)>0$ ). Therefore, $\lambda_{a_{2}}^{*} \leq \lambda_{a_{1}}^{*}$. This shows that $\lambda_{a}^{*}$ is a decreasing function with respect to $a$ and $\lim _{a \rightarrow 0} \lambda_{a}^{*}$ exists, maybe $+\infty$.

We now show (3.2). For any sufficiently small $a>0$, we can choose a proper annulus $A_{a}^{\prime}$ which contains $A_{a}$ with meas $\left(A_{a}^{\prime}\right) \rightarrow 0$ as $a \rightarrow 0$ such that the first eigenfunction $\varphi_{1}^{a}$ with $\left\|\varphi_{1}^{a}\right\|_{\infty}=1$ of the problem

$$
\left\{\begin{array}{lll}
-\Delta \varphi=\sigma \varphi & \text { in } & A_{a}^{\prime} \\
\varphi=0 & \text { on } & \partial A_{a}^{\prime}
\end{array}\right.
$$

satisfies $\frac{1}{2}<m_{a} \leq \varphi_{1}^{a} \leq 1$ on $A_{a}$. Indeed, for any sufficiently small $a>0$, there is a constant $1 \leq M \leq \frac{1}{2 a}$, which is independent of $a$ and will be determined later, such that $1-a M \geq 1 / 2$ (note that $M$ can be chosen to be sufficiently large if $a$ is sufficiently small). Set $A_{a}^{\prime}=\{x: 1-a M<|x|<1+a M\}$. Then $\varphi_{1}:=\varphi_{1}^{a}$ is a positive radial solution of (3.4) and

$$
\begin{aligned}
-\varphi_{1}^{\prime \prime}-\frac{N-1}{r} \varphi_{1}^{\prime} & =\sigma_{1} \varphi_{1}, \quad 1-a M<r<1+a M, \\
\varphi_{1}(1-a M) & =\varphi_{1}(1+a M)=0
\end{aligned}
$$

where $\sigma_{1}:=\sigma_{1}^{a}$ is the first eigenvalue of (3.4) corresponding to the first eigenfunction $\varphi_{1}$. Set $r=1+a \rho$ and $\tilde{\varphi}_{1}(\rho)=\varphi_{1}(r)$. We have that

$$
-\tilde{\varphi}_{1}^{\prime \prime}-\frac{(N-1) a}{1+a \rho} \tilde{\varphi}_{1}^{\prime}=\sigma_{1} a^{2} \tilde{\varphi}_{1}, \quad-M<\rho<M, \quad \tilde{\varphi}_{1}(-M)=\tilde{\varphi}_{1}(M)=0 .
$$

By comparing $\sigma_{1}$ with the first eigenvalue of the eigenvalue problem on the ball $B_{a M}\left(x_{0}\right)$

$$
-\Delta \varphi=\sigma \varphi \quad \text { in } \quad B_{a M}\left(x_{0}\right), \quad \varphi=0 \quad \text { on } \quad \partial B_{a M}\left(x_{0}\right),
$$

where $x_{0}=(1,0, \ldots, 0)$, we find that there is a constant $C:=C(M)>0$ independent of $a$ such that

$$
\sigma_{1} a^{2} \leq C
$$

since $B_{a M}\left(x_{0}\right) \subset A_{a}^{\prime}$. We can now apply the $L^{q}$ estimate and imbeddings to conclude that there is a sequence of $a$, say $a_{n}$, such that $\tilde{\varphi}_{1}^{a_{n}} \rightarrow \psi$ in $C^{1}([-M, M])$, where $\psi(\rho)$ is the positive function satisfying

$$
-\psi^{\prime \prime}=\mu \psi \quad \text { in } \quad(-M, M), \quad \psi(-M)=\psi(M)=0
$$


with $\|\psi\|_{\infty}=1$ and some constant $\mu \in[0, C]$. Clearly $\mu=\left(\frac{\pi}{2 M}\right)^{2}$ and $\psi(\rho)=$ $\cos \left(\frac{\pi \rho}{2 M}\right)$. By a compact and uniqueness argument we have $\varphi_{1}^{a}(1+a \rho) \rightarrow \cos \left(\frac{\pi \rho}{2 M}\right)$ in $C^{1}([-M, M]) \quad$ and $\quad \sigma_{1}^{a} a^{2} \rightarrow\left(\frac{\pi}{2 M}\right)^{2}$ as $a \rightarrow 0^{+}$.

Therefore for sufficiently small $a>0$ and $1-\frac{a M}{3}<r<1+\frac{a M}{3}$, we have

$$
\varphi_{1}^{a}(r)>\frac{1}{2} \text { and } \frac{\pi^{2}}{8 M^{2} a^{2}}<\sigma_{1}^{a}<\frac{\pi^{2}}{2 M^{2} a^{2}} .
$$

Fix an $M \geq 6$. We have $A_{a} \subset A_{a}^{\prime}$ and $\varphi_{1}^{a}>\frac{1}{2}$ on $A_{a}$. Then we see that $\varphi_{1}^{a}$ is a supersolution to (3.1) with

$$
\lambda_{a}=\sigma_{1} \min _{\frac{1}{2} \leq s \leq 1} \frac{s}{f(s)} .
$$

This implies that

$$
\lambda_{a} \leq \lambda_{a}^{*}
$$

Since $\sigma_{1}:=\sigma_{1}^{a} \rightarrow \infty$ as $a \rightarrow 0$, we easily see $\lambda_{a} \rightarrow \infty$ as $a \rightarrow 0$ and hence $\lambda_{a}^{*} \rightarrow \infty$ as $a \rightarrow 0$. Moreover, (3.2) can be obtained from (3.7)-(3.9).

Lemma 3.2. Let $D_{R}=\left\{x \in \mathbb{R}^{N}: R<|x|<R+1\right\}$ with $R$ satisfying (1.2). There exists $\lambda_{R}^{*}>0$ such that problem

$$
\begin{cases}-\Delta u=\lambda f(u) & \text { in } D_{R} \\ u=0 & \text { on } \partial D_{R}\end{cases}
$$

admits no radial solutions for $\lambda>\lambda_{R}^{*}$, one and only one radial solution $u_{R}^{*}$ for $\lambda=\lambda_{R}^{*}$ and exactly two radial solutions $0<\underline{u}_{R}^{\lambda}<\bar{u}_{R}^{\lambda}$ for $0<\lambda<\lambda_{R}^{*}$. Moreover,

$$
\lambda_{R}^{*} \geq C
$$

where $C>0$ is given in (3.2).

Proof. We only need to show (3.11). Making the transformation:

$$
v(\rho)=u(r), \quad \rho=\frac{r}{R},
$$

we see that $v$ satisfies the problem

$$
\begin{cases}-\Delta v=\lambda R^{2} f(v) & \text { in } A_{\frac{1}{R}} \\ v=0 & \text { on } \partial A_{\frac{1}{R}}\end{cases}
$$

It follows from Lemma 3.1 that

$$
\lambda_{R}^{*} R^{2}=\lambda_{\frac{1}{R}}^{*} .
$$

Both (3.13) and (3.2) imply (3.11).

Let $R^{*}$ be a large number which satisfies (1.2). For each $R \geq R^{*}$, we now study the linearized problem at the solution $\left(\lambda, \bar{u}_{R}^{\lambda}\right)$ of (3.10):

$$
\left\{\begin{array}{l}
\Delta h+\lambda f^{\prime}\left(\bar{u}_{R}^{\lambda}\right) h=-\mu h \text { in } D_{R}, \\
h=0 \text { on } \partial D_{R}
\end{array}\right.
$$


It is known from Crandall and Rabinowitz [4] that $i\left(\lambda, \bar{u}_{R}^{\lambda}\right) \geq 1$ and $i_{\text {rad }}\left(\lambda, \bar{u}_{R}^{\lambda}\right) \geq 1$ for $\lambda \in\left(0, \lambda_{R}^{*}\right)$, since $f(s)$ is convex.

Let $\Lambda^{*}=\inf _{R \geq R^{*}} \lambda_{R}^{*}$. It is known from (3.11) that $\Lambda^{*} \geq C$.

Proof of Theorem 1.1 Let $\left(\sigma_{R}^{1}, \phi_{R}^{1}\right)$ be the first eigenvalue and eigenfunction of the problem

$$
\left\{\begin{array}{lll}
-\Delta \phi=\sigma_{R}^{1} \phi & \text { in } & D_{R} \\
\phi=0 & \text { on } & \partial D_{R} .
\end{array}\right.
$$

It is known from Lemma A.1 of [19] that $\sigma_{R}^{1} \rightarrow \pi^{2}$ as $R \rightarrow \infty$. We know from the conditions of $f(s)$ that there exists a constant $A>0$ such that $f(s) \geq A s$ for $s \in[0, \infty)$. Then multiplying $\phi_{R}^{1}$ on both the sides of the equation in $(3.10)$, we see that

$$
\sigma_{R}^{1} \int_{D_{R}} u \phi_{R}^{1} d x \geq \lambda A \int_{D_{R}} u \phi_{R}^{1} d x .
$$

This implies that

$$
\lambda_{R}^{*} \leq \frac{1}{A} \sigma_{R}^{1}
$$

Both (3.15) and (3.11) imply that there is $R_{*}>R^{*}$ such that for $R \geq R_{*}$,

$$
C \leq \lambda_{R}^{*} \leq \frac{10}{A} \pi^{2}
$$

For any $\lambda \in\left(0, \min _{R \geq R_{*}} \lambda_{R}^{*}\right]$, we know that $i\left(\lambda, \underline{u}_{R}\right)=0$ for all $R \geq R_{*}$, since $\underline{u}_{R}$ is the minimal positive solution of (3.10) and hence is stable. Then we claim that there is $C:=C(\lambda)>0$, which is independent of $R$, such that for all $R \geq R_{*}$,

$$
\underline{u}_{R}+\left|\nabla \underline{u}_{R}\right|^{\frac{2}{p+1}} \leq C \operatorname{dist}^{-\frac{2}{p-1}}\left(x, \partial D_{R}\right) \quad \forall x \in D_{R} .
$$

Note that we write $\underline{u}_{R}^{\lambda}$ as $\underline{u}_{R}$.

Assume that (3.17) fails. Then there exist sequences $\left\{R_{k}\right\},\left\{\underline{u}_{k}\right\}\left(\equiv\left\{\underline{u}_{R_{k}}\right\}\right)$, $y_{k} \in D_{R_{k}}$, such that $\underline{u}_{k}$ solves the problem

$$
\begin{cases}-\Delta u=\lambda f(u) & \text { in } D_{R_{k}} \\ u=0 & \text { on } \partial D_{R_{k}}\end{cases}
$$

and the functions

$$
M_{k}(x):=\underline{u}_{k}^{\frac{p-1}{2}}+\left|\nabla \underline{u}_{k}\right|^{\frac{p-1}{p+1}}, \quad k=1,2, \ldots
$$

satisfy

$$
M_{k}\left(y_{k}\right)>2 k \operatorname{dist}^{-1}\left(y_{k}, \partial D_{R_{k}}\right) .
$$

By a doubling lemma (see Lemma 5.1) of [23] we see that there exists $x_{k} \in D_{R_{k}}$ such that

$$
M_{k}\left(x_{k}\right) \geq M_{k}\left(y_{k}\right), \quad M_{k}\left(x_{k}\right)>2 k \operatorname{dist}^{-1}\left(x_{k}, \partial D_{R_{k}}\right)
$$

and

$$
M_{k}(z) \leq 2 M_{k}\left(x_{k}\right), \quad\left|z-x_{k}\right| \leq k M_{k}^{-1}\left(x_{k}\right)
$$


Now we rescale $\underline{u}_{k}$ by setting

$$
w_{k}(y):=\tau_{k}^{2 /(p-1)} \underline{u}_{k}\left(x_{k}+\tau_{k} y\right), \quad|y| \leq k \quad \text { with } \quad \tau_{k}=M_{k}^{-1}\left(x_{k}\right) .
$$

Then $w_{k}$ solves

$$
\begin{gathered}
-\Delta_{y} w_{k}(y)=f_{k}\left(w_{k}(y)\right):=\tau_{k}^{\frac{2 p}{p-1}} f\left(\tau_{k}^{-\frac{2}{p-1}} w_{k}(y)\right), \quad|y| \leq k, \\
{\left[w_{k}^{\frac{p-1}{2}}+\left|\nabla w_{k}\right|^{\frac{p-1}{p+1}}\right](0)=\tau_{k} M_{k}\left(x_{k}\right)=1}
\end{gathered}
$$

and

$$
\left[w_{k}^{\frac{p-1}{2}}+\left|\nabla w_{k}\right|^{\frac{p-1}{p+1}}\right](y) \leq 2, \quad|y| \leq k .
$$

Arguments similar to those in the proof of Theorem 2.1 of [23] imply that some subsequence of $\left\{w_{k}\right\}$ converges in $C_{l o c}^{1}\left(\mathbb{R}^{N}\right)$ to a function $w$ and $w$ is a positive solution of

$$
-\Delta w=\lambda w^{p}, \quad y \in \mathbb{R}^{N} .
$$

Moreover, $w$ and $|\nabla w|$ are bounded in $\mathbb{R}^{N}$. This is a contradiction provided $1<p<\frac{N+2}{N-2}$. On the other hand, since $i\left(\lambda, \underline{u}_{k}\right)=0$ for all $k$, we see that $i\left(\lambda, w_{k}\right)=i\left(\lambda, \underline{u}_{k}\right)=0$ for all $k$ and hence $i(\lambda, w)=0$ (see the proof of step 1 in the proof of Theorem 1.2 below). This implies that $w$ is a regular stable positive solution of (3.19) and contradictions for $p=\frac{N+2}{N-2}$ and $\frac{N+2}{N-2}<p<p_{c}(N)$. It is known from $[9,10,12]$ that regular stable positive solution of (3.19) does not exist for $p=\frac{N+2}{N-2}$; it is also known from $[8,9,11,12]$ that $i(\lambda, w)=\infty$ provided $\frac{N+2}{N-2}<p<p_{c}(N)$. Therefore, (3.17) holds.

We now use the blow-up argument again to obtain an upper bound for $\underline{u}_{R}$. We have that there exists $C:=C(\lambda)>0$ independent of $R$ such that for all $R>R_{*}$ that

$$
\underline{u}_{R} \leq C \quad \text { in } \quad D_{R}
$$

On the contrary, there is a sequence $\left\{R_{k}\right\}$ with $R_{k} \rightarrow \infty$ as $k \rightarrow \infty$ such that $\underline{u}_{R_{k}}\left(x_{k}\right) \rightarrow \infty$ as $k \rightarrow \infty$, where $\underline{u}_{R_{k}}\left(x_{k}\right)=\max _{D_{R_{k}}} \underline{u}_{R_{k}}$. Denote $C_{k}=\underline{u}_{R_{k}}\left(x_{k}\right)(\rightarrow \infty$ as $k \rightarrow \infty)$. Setting

$$
y=C_{k}^{\frac{p-1}{2}}\left(x-\eta_{k}\right), \quad \tilde{u}_{k}(y)=\frac{\underline{u}_{R_{k}}(x)}{C_{k}},
$$

where $\eta_{k} \in \partial D_{R_{k}}$ such that $\operatorname{dist}\left(x_{k}, \eta_{k}\right)=\operatorname{dist}\left(x_{k}, \partial D_{R_{k}}\right)$, we see that $\tilde{u}_{k}$ satisfies the problem

$$
\begin{cases}-\Delta_{y} \tilde{u}_{k}=\lambda \frac{f\left(C_{k} \tilde{u}_{k}\right)}{C_{k}^{p}} & \text { in } \Omega_{k}, \\ \tilde{u}_{k}=0 & \text { on } \partial \Omega_{k},\end{cases}
$$

where $\Omega_{k}=\left\{y=C_{k}^{\frac{p-1}{2}}\left(x-\eta_{k}\right), x \in D_{R_{k}}\right\}$. Moreover, it is known from (3.17) that there is $C>0$ independent of $k$ such that

$$
C_{k}^{\frac{p-1}{2}}\left(x_{k}-\eta_{k}\right) \leq C .
$$


Using elliptic $L^{p}$ estimates and standard imbeddings, we deduce that some subsequence of $\left\{\tilde{u}_{k}\right\}$ converges in $C_{l o c}^{1}(\Gamma)$ to a nonnegative (classical) solution $\tilde{u}$ of

$$
\begin{cases}-\Delta \tilde{u}=\lambda \tilde{u}^{p}, & \text { in } \Gamma, \\ \tilde{u}=0, & \text { on } \partial \Gamma,\end{cases}
$$

where $\Gamma$ is a half space of $\mathbb{R}^{N}$, say $\Gamma=\left\{y=\left(y_{1}, \ldots, y_{N}\right), y_{N}>0\right\}$. Moreover, $\tilde{u}(\tilde{y})=\max _{y \in \Gamma} \tilde{u}(y)=1$ and $\operatorname{dist}(\tilde{y}, 0) \leq C$, where $\tilde{y}=\lim _{k \rightarrow \infty} C_{k}^{\frac{p-1}{2}}\left(x_{k}-\right.$ $\left.\eta_{k}\right) \in \Gamma$. This is impossible since it is known from Theorem 2 of [7] that $\frac{\partial \tilde{u}(y)}{\partial y_{N}}>0$ for $y \in \bar{\Gamma}$. This implies that (3.20) holds.

We now show that

$$
\underline{u}_{R}(R+t) \rightarrow \underline{u}_{0}(t) \quad \text { uniformly for } t \in[0,1] \quad \text { as } \quad R \rightarrow \infty,
$$

where $\underline{u}_{0}$ is the minimal solution of the problem

$$
\left\{\begin{array}{l}
-u_{0}^{\prime \prime}=\lambda f\left(u_{0}\right) \quad \text { in } \quad(0,1), \\
u_{0}(0)=u_{0}(1)=0 .
\end{array}\right.
$$

Set $\tilde{u}_{R}(t)=\underline{u}_{R}(t+R)$. We see that for all $R \geq R_{*}$,

$$
\begin{aligned}
\lambda \int_{0}^{1} f\left(\tilde{u}_{R}(t)\right) d t & =\lambda \int_{R}^{R+1} f\left(\underline{u}_{R}\right) d r \\
& \leq \frac{\lambda}{R^{N-1}} \int_{R}^{R+1} r^{N-1} f\left(\underline{u}_{R}\right) d r \\
& \leq C
\end{aligned}
$$

where $C>0$ is independent of $R$ (we use (3.20) here).

Multiplying (1.1) by $\underline{u}_{R}$ and integrating, we see from (3.24) that

$$
\int_{R}^{R+1} r^{N-1}\left(\underline{u}_{R}^{\prime}\right)^{2} d r=\lambda \int_{R}^{R+1} r^{N-1} f\left(\underline{u}_{R}\right) d r \leq C R^{N-1} .
$$

This implies that

$$
R^{N-1}\left(\int_{0}^{1}\left(\tilde{u}_{R}^{\prime}\right)^{2} d t\right) \leq \int_{R}^{R+1} r^{N-1}\left(\underline{u}_{R}^{\prime}\right)^{2} d r \leq C R^{N-1}
$$

and hence

$$
\int_{0}^{1}\left(\tilde{u}_{R}^{\prime}\right)^{2} d t \leq C
$$

Observe that $\tilde{u}_{R}$ satisfies the problem

$$
\left\{\begin{array}{l}
-\tilde{u}_{R}^{\prime \prime}-\frac{N-1}{t+R} \tilde{u}_{R}^{\prime}=\lambda f\left(\tilde{u}_{R}\right) \text { in }(0,1), \\
\tilde{u}_{R}(0)=\tilde{u}_{R}(1)=0 .
\end{array}\right.
$$

Therefore, since by $(3.25) \tilde{u}_{R}$ is bounded in $H_{0}^{1}(0,1)$, it is also bounded in $C^{1}(0,1)$. Consequently there is a sequence $\left\{R_{k}\right\}$ such that $\tilde{u}_{R_{k}} \rightarrow \hat{u}_{0}$ uniformly for $t \in[0,1]$ as $k \rightarrow \infty$, where $\hat{u}_{0}$ satisfies (3.23). Let us show $\hat{u}_{0}=\underline{u}_{0}$. We know that

$$
i\left(\lambda, \underline{u}_{R_{k}}\right)=i_{\text {rad }}\left(\lambda, \underline{u}_{R_{k}}\right)=i\left(\lambda, \tilde{u}_{R_{k}}\right)=i_{\text {rad }}\left(\lambda, \tilde{u}_{R_{k}}\right)=0 \text { for all } \quad R_{k} \geq R_{*} .
$$


Then $i\left(\lambda, \hat{u}_{0}\right)=0$ (note that $\left.i\left(\lambda, \hat{u}_{0}\right)=i_{\text {rad }}\left(\lambda, \hat{u}_{0}\right)\right)$ and hence $\hat{u}_{0} \equiv \underline{u}_{0}$. By a compact and uniqueness argument we have that $\underline{u}_{R}(R+t) \rightarrow \underline{u}_{0}(t)$ uniformly for $t \in[0,1]$ as $R \rightarrow \infty$. This also implies $\min _{R \geq R_{*}} \lambda_{R}^{*} \leq \lambda_{0}^{*}$.

We now show (1.6). On the contrary, there is a sequence $\left\{R_{k}\right\}$ with $R_{k} \rightarrow \infty$ as $k \rightarrow \infty$ such that $\lambda_{R_{k}}^{*} \rightarrow \hat{\lambda} \neq \lambda_{0}^{*}$ as $k \rightarrow \infty$. It follows from (3.16) that $0<\hat{\lambda}<\infty$. Arguments similar to those in the proof of (3.22) imply that $u_{R_{k}}^{*}\left(R_{k}+t\right) \rightarrow \hat{u}(t)$ uniformly for $t \in[0,1]$ as $k \rightarrow \infty$, where $\hat{u}(t)$ solves the ODE:

$$
\left\{\begin{array}{l}
-\hat{u}^{\prime \prime}=\hat{\lambda} f(\hat{u}) \quad \text { in }(0,1), \\
\hat{u}(0)=\hat{u}(1)=0 .
\end{array}\right.
$$

On the other hand, we know that for all $k$, the first eigenvalues $\mu_{1}\left(u_{R_{k}}^{*}\right)$ of the problem

$$
\left\{\begin{array}{l}
\phi^{\prime \prime}(r)+\frac{N-1}{r} \phi^{\prime}(r)+\lambda_{R_{k}}^{*} f^{\prime}\left(u_{R_{k}}^{*}\right) \phi=-\mu \phi \text { in }\left(R_{k}, R_{k}+1\right), \\
\left.\phi\left(R_{k}\right)\right)=\phi\left(R_{k}+1\right)=0
\end{array}\right.
$$

equal to 0 for all $k$. This implies that for any $k$, there is a $\phi_{k}(r)>0$ for $r \in\left(R_{k}, R_{k}+1\right)$ and $\left\|\phi_{k}\right\|_{L^{\infty}\left(R_{k}, R_{k}+1\right)}=1$ such that

$$
\left\{\begin{array}{l}
\phi_{k}^{\prime \prime}(r)+\frac{N-1}{r} \phi_{k}^{\prime}(r)+\lambda_{R_{k}}^{*} f^{\prime}\left(u_{R_{k}}^{*}\right) \phi_{k}=0 \text { in }\left(R_{k}, R_{k}+1\right), \\
\left.\phi_{k}\left(R_{k}\right)\right)=\phi_{k}\left(R_{k}+1\right)=0 .
\end{array}\right.
$$

Set

$$
\tilde{\phi}_{k}(t)=\phi_{k}(r), \quad r=R_{k}+t .
$$

We see that $\tilde{\phi}_{k}>0$ in $(0,1)$ and $\left\|\tilde{\phi}_{k}\right\|_{L^{\infty}(0,1)}=1$, which satisfies

$$
\left\{\begin{array}{l}
\tilde{\phi}_{k}^{\prime \prime}(t)+\frac{N-1}{R_{k}+t} \tilde{\phi}_{k}^{\prime}(t)+\lambda_{R_{k}}^{*} f^{\prime}\left(u_{R_{k}}^{*}\left(R_{k}+t\right)\right) \tilde{\phi}_{k}(t)=0 \text { in }(0,1), \\
\tilde{\phi}_{k}(0)=\tilde{\phi}_{k}(1)=0 .
\end{array}\right.
$$

The regularity arguments as the above imply that there is a subsequence of $\left\{\tilde{\phi}_{k}\right\}$ (still denoted by $\left.\left\{\tilde{\phi}_{k}\right\}\right)$ such that

$$
\tilde{\phi}_{k} \rightarrow \tilde{\phi} \text { in } C^{1}(0,1) \text { as } k \rightarrow \infty
$$

and $\tilde{\phi}>0$ in $(0,1)$ with $\|\tilde{\phi}\|_{L^{\infty}(0,1)}=1$, which satisfies the problem

$$
\left\{\begin{array}{l}
\tilde{\phi}^{\prime \prime}(t)+\hat{\lambda} f^{\prime}(\hat{u}) \tilde{\phi}=0 \text { in }(0,1), \\
\tilde{\phi}(0)=\tilde{\phi}(1)=0 .
\end{array}\right.
$$

This implies that $\hat{\lambda}=\lambda_{0}^{*}$ and $\hat{u} \equiv u_{0}^{*}$ and contradicts our assumption $\hat{\lambda} \neq \lambda_{0}^{*}$. Therefore, (1.6) holds and for any $\lambda \in\left(0, \lambda_{0}^{*}\right), \underline{u}_{R}(R+t) \rightarrow \underline{u}_{0}(t)$ uniformly for $t \in[0,1]$ as $R \rightarrow \infty, u_{R}^{*}(R+t) \rightarrow u_{0}^{*}(t)$ uniformly for $t \in[0,1]$ as $R \rightarrow \infty$. It follows from (3.13) that

$$
\lim _{R \rightarrow \infty} \frac{\lambda_{\frac{1}{R}}^{*}}{R^{2}}=\lambda_{0}^{*}
$$

This also implies that

$$
\lim _{a \rightarrow 0^{+}} a^{2} \lambda_{a}^{*}=\lambda_{0}^{*}
$$

where $\lambda_{a}^{*}$ is as in Lemma 3.1. 
Proof of Theorem 1.2 Since $\lambda \in\left(0, \lambda_{0}^{*}\right)$ and $\lim _{R \rightarrow \infty} \lambda_{R}^{*}=\lambda_{0}^{*}$, we can choose $R_{*}$ (given above) such that $\lambda \in\left(0, \lambda_{R}^{*}\right)$ provided $R \geq R_{*}$.

Suppose that the conclusion of Theorem 1.2 does not hold, we see that there exists an integer $\theta \geq 1$ independent of $R$ such that

$$
i\left(\lambda, \bar{u}_{R}^{\lambda}\right) \leq \theta \quad \forall R \geq R_{*}
$$

For convenience, we write $\bar{u}_{R}^{\lambda}$ as $\bar{u}_{R}$ in the following.

The proof can be divided into several steps:

Step 1. We show that there is $C>0$ independent of $R$ such that for any $R \geq R_{*}$,

$$
\bar{u}_{R}+\left|\nabla \bar{u}_{R}\right|^{\frac{2}{p+1}} \leq C \text { dist }^{-\frac{2}{p-1}}\left(x, \partial D_{R}\right) \quad \forall x \in D_{R} .
$$

The proof of (3.29) is similar to that of (3.17) provided that (3.28) holds. Indeed, we use the blow-up argument as in the proof of (3.17). If we define

$$
\tilde{M}_{k}(x):=\bar{u}_{k}^{\frac{p-1}{2}}+\left|\nabla \bar{u}_{k}\right|^{\frac{p-1}{p+1}}, \quad k=1,2, \ldots
$$

and

$$
\tilde{w}_{k}(y):=\tilde{\tau}_{k}^{2 /(p-1)} \bar{u}_{k}\left(x_{k}+\tilde{\tau}_{k} y\right), \quad|y| \leq k \quad \text { with } \quad \tilde{\tau}_{k}=\tilde{M}_{k}^{-1}\left(x_{k}\right),
$$

we see that $\tilde{w}_{k}(y)$ satisfies the equation

$$
\begin{gathered}
-\Delta_{y} \tilde{w}_{k}(y)=f_{k}\left(\tilde{w}_{k}(y)\right):=\tilde{\tau}_{k}^{\frac{2 p}{p-1}} f\left(\tilde{\tau}_{k}^{-\frac{2}{p-1}} \tilde{w}_{k}(y)\right), \quad|y| \leq k, \\
{\left[\tilde{w}_{k}^{\frac{p-1}{2}}+\left|\nabla \tilde{w}_{k}\right|^{\frac{p-1}{p+1}}\right](0)=\tilde{\tau}_{k} \tilde{M}_{k}\left(x_{k}\right)=1}
\end{gathered}
$$

and

$$
\left[\tilde{w}_{k}^{\frac{p-1}{2}}+\left|\nabla \tilde{w}_{k}\right|^{\frac{p-1}{p+1}}\right](y) \leq 2, \quad|y| \leq k .
$$

On the other hand, we see that the first eigenvalue

$$
\begin{aligned}
\mu_{1}^{(k)} & =\inf _{\varphi \in H_{0}^{1}\left(D_{R_{k}}\right), \varphi \neq 0} \frac{\int_{D_{R_{k}}}\left[\left|\nabla_{x} \varphi\right|^{2}-\lambda f^{\prime}\left(\bar{u}_{k}\right) \varphi^{2}\right] d x}{\int_{D_{R_{k}}} \varphi^{2} d x} \\
& =\inf _{\psi \in H_{0}^{1}\left(\tilde{D}_{R_{k}}\right), \psi \neq 0} \frac{\int_{\tilde{D}_{R_{k}}}\left[\left|\nabla_{y} \psi\right|^{2}-\lambda f_{k}^{\prime}\left(\tilde{w}_{k}(y)\right) \psi^{2}\right] d y}{\tilde{\tau}_{k}^{2} \int_{\tilde{D}_{R_{k}}} \psi^{2} d y},
\end{aligned}
$$

where $\tilde{D}_{R_{k}}=\left\{y: x_{k}+\tilde{\tau}_{k} y \in D_{R_{k}}\right\}, \psi(y):=\varphi(x)$ and $x=x_{k}+\tilde{\tau}_{k} y$. This implies that

$$
\tilde{\mu}_{1}^{(k)}=\tilde{\tau}_{k}^{2} \mu_{1}^{(k)}
$$

where

$$
\tilde{\mu}_{1}^{(k)}=\inf _{\psi \in H_{0}^{1}\left(\tilde{D}_{R_{k}}\right), \psi \neq 0} \frac{\int_{\tilde{D}_{R_{k}}}\left[\left|\nabla_{y} \psi\right|^{2}-\lambda f_{k}^{\prime}\left(\tilde{w}_{k}\right) \psi^{2}\right] d y}{\int_{\tilde{D}_{R_{k}}} \psi^{2} d y} .
$$


By the fact that

$$
\mu_{j}^{(k)}=\inf _{\mathcal{A} \subset H_{0}^{1}\left(D_{R_{k}}\right), \operatorname{dim} \mathcal{A}=j \varphi \in \mathcal{A}, \varphi \neq 0} \frac{\int_{D_{R_{k}}}\left[\left|\nabla_{x} \varphi\right|^{2}-\lambda f^{\prime}\left(\bar{u}_{k}\right) \varphi^{2}\right] d x}{\int_{D_{R_{k}}} \varphi^{2} d x},
$$

we also see that

$$
\tilde{\mu}_{j}^{(k)}=\tilde{\tau}_{k}^{2} \mu_{j}^{(k)},
$$

where

$$
\tilde{\mu}_{j}^{(k)}=\inf _{\mathcal{B} \subset H_{0}^{1}\left(\tilde{D}_{R_{k}}\right), \operatorname{dim} \mathcal{B}=j} \max _{\psi \in \mathcal{B}, \psi \neq 0} \frac{\int_{\tilde{D}_{R_{k}}}\left[\left|\nabla_{y} \psi\right|^{2}-\lambda f_{k}^{\prime}\left(\tilde{w}_{k}\right) \psi^{2}\right] d y}{\int_{\tilde{D}_{R_{k}}} \psi^{2} d y} .
$$

This and (3.28) imply that

$$
i\left(\lambda, \tilde{w}_{k}\right) \leq \theta .
$$

Therefore, the maximal dimension of all subspaces $X$ of $C_{0}^{1}\left(\tilde{D}_{R_{k}}\right)$ such that

$$
\int_{\tilde{D}_{R_{k}}}\left[\left|\nabla_{y} \psi\right|^{2}-\lambda f_{k}^{\prime}\left(\tilde{w}_{k}\right) \psi^{2}\right] d y<0 \quad \forall \psi \in X \backslash\{0\}
$$

is not bigger than $\theta$. Note that $\tilde{\tau}_{k}^{2} \rightarrow 0$ as $k \rightarrow \infty$.

On the other hand, arguments similar to those in the proof of (3.17) imply that $\left\{\tilde{w}_{k}\right\}$ converges in $C_{l o c}^{1}\left(\mathbb{R}^{N}\right)$ to a (classical) solution $\tilde{w}$ of the equation

$$
-\Delta \tilde{w}=\lambda \tilde{w}^{p} \text { in } \mathbb{R}^{N} \text {. }
$$

Moreover $\left[\tilde{w}^{\frac{p-1}{2}}+|\nabla \tilde{w}|^{\frac{p-1}{p+1}}\right](0)=1$. This is impossible for $1<p<\frac{N+2}{N-2}$. For $\frac{N+2}{N-2}<p<p_{c}(N)$, it follows from (3.32) that the maximal dimension of all subspaces $X$ of $C_{0}^{1}\left(\mathbb{R}^{N}\right)$ such that

$$
\int_{\mathbb{R}^{N}}\left[\left|\nabla_{y} \psi\right|^{2}-\lambda p \tilde{w}^{p-1} \psi^{2}\right] d y<0 \quad \forall \psi \in X \backslash\{0\}
$$

is not bigger than $\theta$ and thus

$$
i(\lambda, \tilde{w}) \leq \theta .
$$

Note that for each $y \in \mathbb{R}^{N}, f_{k}^{\prime}\left(\tilde{w}_{k}(y)\right) \rightarrow p \tilde{w}^{p-1}(y)$. It is known from $[8-10,12]$ that $i(\lambda, \tilde{w})=\infty$ provided $\frac{N+2}{N-2}<p<p_{c}(N)$. This contradicts to (3.34). These contradictions imply that (3.29) holds. (We can not obtain (3.29) for $p=\frac{N+2}{N-2}$, since in this case, (1.3) admits solutions with finite Morse index.) Then the blow-up argument as in the proof of (3.20) implies that there is $C:=C(\lambda)$ independent of $R$ such that for $R \geq R_{*}$,

$$
\bar{u}_{R} \leq C \text { in } D_{R}
$$

provided that (3.28) holds.

Step 2. We show that $\bar{u}_{R}(R+t) \rightarrow \bar{u}_{0}^{\lambda}(t)$ uniformly for $t \in[0,1]$ as $R \rightarrow \infty$, where $\bar{u}_{0}^{\lambda}$ is the solution on the upper solution branch of the problem

$$
\left\{\begin{array}{l}
-u_{0}^{\prime \prime}=\lambda f\left(u_{0}\right) \quad \text { in }(0,1), \\
u_{0}(0)=u_{0}(1)=0 .
\end{array}\right.
$$


The proof of this step is similar to that of (3.22). To guarantee that the limit is $\bar{u}_{0}^{\lambda}$, we need to notice that $i_{\text {rad }}\left(\lambda, \bar{u}_{R}\right) \geq 1$ for all $R \geq R_{*}$.

Step 3. We show that if $\hat{\mu}_{1}(R)$ is the first eigenvalue of the operator $\hat{L}_{R}$ defined by $\hat{L}_{R}: H^{2}(R, R+1) \cap H_{0}^{1}(R, R+1) \rightarrow L^{2}(R, R+1)$,

$$
\hat{L}_{R}(w)=r^{2}\left(-w^{\prime \prime}-\frac{1}{r} w^{\prime}-\lambda f^{\prime}\left(\bar{u}_{R}\right) w\right) \quad r \in(R, R+1) .
$$

Then

$$
\hat{\mu}_{1}(R)=\sigma_{1} R^{2}+o\left(R^{2}\right) \text { as } R \rightarrow \infty
$$

where $\sigma_{1}<0$ is the first eigenvalue for the problem

$$
\left\{\begin{array}{l}
-w^{\prime \prime}-\lambda f^{\prime}\left(\bar{u}_{0}^{\lambda}\right) w=\sigma w \quad \text { in }(0,1) \\
w(0)=w(1)=0
\end{array}\right.
$$

where $\bar{u}_{0}^{\lambda}$ is as in step 2 .

The proof of this step is standard (see Lemma 3.2 of [1] and Proposition 3.2 of [14]). The eigenvalue $\hat{\mu}_{1}(R)$ can be characterized as

$$
\hat{\mu}_{1}(R)=\inf _{w \in H_{0}^{1}(R, R+1), w \neq 0} \frac{\int_{R}^{R+1} r^{N-1}\left(w^{\prime}\right)^{2} d r-\int_{R}^{R+1} \lambda r^{N-1} f^{\prime}\left(\bar{u}_{R}\right) w^{2} d r}{\int_{R}^{R+1} r^{N-3} w^{2} d r} .
$$

To estimate $\hat{\mu}_{1}(R)$ let us consider a function $\hat{\phi} \in C_{0}^{\infty}(0,1), \hat{\phi} \geq 0$ and set $\phi(r)=\hat{\phi}(r-R)$. Then

$$
\begin{aligned}
\hat{\mu}_{1}(R) & \leq \frac{\int_{R}^{R+1} r^{N-1}\left(\phi^{\prime}\right)^{2} d r-\int_{R}^{R+1} \lambda r^{N-1} f^{\prime}\left(\bar{u}_{R}\right) \phi^{2} d r}{\int_{R}^{R+1} r^{N-3} \phi^{2} d r} \\
& =\frac{\int_{0}^{1}(R+t)^{N-1}\left(\hat{\phi}^{\prime}\right)^{2} d t-\int_{0}^{1} \lambda(R+t)^{N-1} f^{\prime}\left(\tilde{u}_{R}\right) \hat{\phi}^{2} d t}{\int_{0}^{1}(R+t)^{N-3} \hat{\phi}^{2} d t} \\
& \leq C R^{2} .
\end{aligned}
$$

In order to get the reverse inequality let us denote by $w_{1, R}$ the eigenfunction associated to $\hat{\mu}_{1}(R)$ with $\left\|w_{1, R}\right\|_{L^{\infty}\left(D_{R}\right)}=1$. Inserting $w_{1, R}$ into (3.39) we have, for a positive constant $C>0$,

$$
\begin{aligned}
\hat{\mu}_{1}(R) & =\frac{\int_{R}^{R+1} r^{N-1}\left(w_{1, R}^{\prime}\right)^{2} d r-\int_{R}^{R+1} \lambda r^{N-1} f^{\prime}\left(\bar{u}_{R}\right) w_{1, R}^{2} d r}{\int_{R}^{R+1} r^{N-3} w_{1, R}^{2} d r} \\
& \geq \frac{-\lambda K \int_{R}^{R+1} r^{N-1} w_{1, R}^{2} d r}{\int_{R}^{R+1} r^{N-3} w_{1, R}^{2} d r} \\
& \geq-C R^{2} .
\end{aligned}
$$

since we know from step 1 and the conditions of $f$ that $0<f^{\prime}\left(\bar{u}_{R}\right) \leq K$.

Now we define $\tilde{w}_{1, R}(t)=w_{1, R}(t+R)$ in $(0,1)$, we see that $\tilde{w}_{1, R}$ satisfies the problem

$$
\left\{\begin{array}{l}
-\tilde{w}_{1, R}^{\prime \prime}-\frac{N-1}{t+R} \tilde{w}_{1, R}^{\prime}-\lambda f^{\prime}\left(\tilde{u}_{R}\right) \tilde{w}_{1, R}=\hat{\mu}_{1}(R) \frac{\tilde{w}_{1, R}}{(t+R)^{2}} \text { in }(0,1) \\
\tilde{w}_{1, R}(0)=\tilde{w}_{1, R}(1)=0
\end{array}\right.
$$


It is known from (3.40) and (3.41) that $\left|\frac{\hat{\mu}_{1}(R)}{R^{2}}\right| \leq C$ for $R$ sufficiently large. Since $\left\|\tilde{w}_{1, R}\right\|_{\infty}=1$, the regularity argument as the above implies that there is a sequence $\left\{R_{k}\right\}$ with $R_{k} \rightarrow \infty$ as $k \rightarrow \infty$ such that $\tilde{w}_{1, R_{k}} \rightarrow \xi_{1} \geq 0$ uniformly in $[0,1]$ as $k \rightarrow \infty$, and $\xi_{1}$ with $\left\|\xi_{1}\right\|_{L^{\infty}(0,1)}=1$ is a solution to the problem

$$
\left\{\begin{array}{l}
-\xi_{1}^{\prime \prime}-\lambda f^{\prime}\left(\bar{u}_{0}^{\lambda}\right) \xi_{1}=\sigma_{1} \xi_{1} \text { in }(0,1) \\
\xi_{1}(0)=\xi_{1}(1)=0
\end{array}\right.
$$

where $\bar{u}_{0}^{\lambda}$ is as in step 2 and $\sigma_{1}=\lim _{k \rightarrow \infty} \frac{\hat{\mu}_{1}\left(R_{k}\right)}{R_{k}^{2}}$. The strong maximum principle implies that $\xi_{1}>0$ in $(0,1)$. Hence, $\sigma_{1}$ is the first eigenvalue of the problem (3.38) and $\xi_{1}$ is the corresponding eigenfunction. The uniqueness of the first eigenvalue and corresponding eigenfunction of (3.38) implies that

$$
\lim _{R \rightarrow \infty} \frac{\hat{\mu}_{1}(R)}{R^{2}}=\sigma_{1}<0, \quad \tilde{w}_{1, R} \rightarrow \xi_{1} \quad \text { uniformly in } \quad[0,1] \text { as } R \rightarrow \infty .
$$

Then we have, with a change of variable, from (3.41):

$$
\hat{\mu}_{1}(R)=\frac{\int_{0}^{1}(t+R)^{N-1}\left(\tilde{w}_{1, R}^{\prime}\right)^{2} d t-\int_{0}^{1} \lambda(t+R)^{N-1} f^{\prime}\left(\tilde{u}_{R}\right) \tilde{w}_{1, R}^{2} d t}{\int_{0}^{1}(t+R)^{N-3} \tilde{w}_{1, R}^{2} d t}
$$

and, passing to the limit as $R \rightarrow \infty$,

$$
\frac{\hat{\mu}_{1}(R)}{R^{2}}=\frac{\int_{0}^{1}\left(\xi_{1}^{\prime}\right)^{2} d t-\lambda \int_{0}^{1} f^{\prime}\left(\bar{u}_{0}^{\lambda}\right) \xi_{1}^{2} d t}{\int_{0}^{1} \xi_{1}^{2} d t}+o(1) .
$$

From this and (3.43) we obtain (3.37).

Step 4. We complete the proof of this theorem.

It is known from Lemma 2.2 that $\tilde{\mu}(R, k)=\hat{\mu}_{1}(R)+\lambda_{k}\left(=\hat{\mu}_{1}(R)+k(k+\right.$ $N-2)), k=0,1,2, \ldots$ are eigenvalues of the operator $\tilde{L}_{\bar{u}_{R}^{\lambda}}$. Note that $\bar{u}_{R}^{\lambda}$ is nondegenerate in the space $H_{0, \text { rad }}^{1}\left(D_{R}\right)$. Since step 3 implies that $\hat{\mu}_{1}(R) \rightarrow-\infty$ as $R \rightarrow \infty$, we see that there are infinitely many negative $\tilde{\mu}(R, k)$ as $R \rightarrow \infty$. Therefore, Lemma 2.1 implies that

$$
i\left(\lambda, \bar{u}_{R}\right) \rightarrow+\infty \text { as } R \rightarrow \infty .
$$

This contradicts to (3.28) and completes the proof of this theorem.

Corollary 3.3. There exists a sequence $\left\{R_{k}\right\}$ with $R_{k} \geq R_{*}$ and $R_{k} \rightarrow+\infty$ as $k \rightarrow \infty$ such that for each $k, \bar{u}_{R_{k}}^{\lambda}$ is degenerate.

Proof. It is known from Theorem 1.2 that there exists a sequence $\left\{R_{j}\right\}$ with $R_{j} \geq R_{*}$ and $R_{j} \rightarrow \infty$ such that $i\left(\lambda, \bar{u}_{R_{j}}^{\lambda}\right) \rightarrow \infty$ as $j \rightarrow \infty$. We can choose a subsequence of $\left\{R_{j}\right\}$ (still denoted by $\left\{R_{j}\right\}$ ) such that for any $j$,

$$
i\left(\lambda, \bar{u}_{R_{j}}^{\lambda}\right)<i\left(\lambda, \bar{u}_{R_{j+1}}^{\lambda}\right) .
$$

By the continuity of the eigenvalues of $L_{\bar{u}_{R}^{\lambda}}$ with respect to $R$, we easily see that for each $j$, there exists $k=k(j)$ such that $R_{k} \in\left[R_{j}, R_{j+1}\right]$ and $\bar{u}_{R_{k}}^{\lambda}$ is degenerate. It is known from Lemma 2.3 that $R_{k}$ can be determined from $\hat{\mu}_{1}\left(R_{k}\right)+\lambda_{k}=0$. This implies that the conclusion of this corollary holds. 
Remark 3.4. The conclusions of Theorem 1.1 hold for the case $N \geq 3$ and $p=\frac{N+2}{N-2}$. We notice that the conclusions of Theorem 1.2 also hold for the case $p=\frac{N+2}{N-2}$ if we have that there is $C>0$ independent of $R$ such that

$$
\left\|\bar{u}_{R}\right\|_{\infty} \leq C .
$$

To obtain (3.44), we need

$$
\tilde{\tau}_{k}^{2} \mu_{1}^{(k)} \rightarrow 0 \text { ask } \rightarrow \infty
$$

in the proof of Theorem 1.2. It is known from (3.30) that $\tilde{\mu}_{j}^{(k)}=\tilde{\tau}_{k}^{2} \mu_{j}^{(k)}$. Note that $\tilde{\tau}_{k}^{2} \rightarrow 0$ as $k \rightarrow \infty$. If we have (3.45), we will see that the $\tilde{w}$ in the proof of Theorem 1.2 is stable. This is impossible, since $\tilde{w}$ is a positive entire solution of (1.3) with $p=\frac{N+2}{N-2}$. (We know that the equation (1.3) does not admit a stable solution.) This implies that we can obtain (3.29) and hence we can obtain (3.44).

Remark 3.5. We still do not know the related results for the case $N \geq 11$ and $p \geq p_{c}(N)$, since we can not obtain the uniform boundedness of $\left\{\left\|\underline{u}_{R}\right\|_{\infty}\right\}$ and $\left\{\left\|\bar{u}_{R}\right\|_{\infty}\right\}$ for large $R$. The blow-up argument as in the proof of Theorems 1.1 and 1.2 can not be used to discuss the case $p \geq p_{c}(N)$, since in this case, the Eq. (1.3) admits positive stable entire solutions.

\section{Bifurcation results}

In this section we obtain the nonradial bifurcation from the radial solution $\bar{u}_{R}$ by showing that there is a change in the Leray-Schauder degree of certain associated maps as $R$ crosses $R_{k}$. Where $R_{k}$ is given in Corollary 3.3. The main idea is similar to those in [14].

Let $\alpha, \beta \in \mathbb{R}$ such that $0<\alpha<\beta$, and let $D:=\left\{x \in \mathbb{R}^{N}, \alpha<|x|<\beta\right\}$. For any $R \in(1, \infty)$, let

$$
h_{R}: D_{R} \rightarrow D
$$

be the diffeomorphism that maps $D_{R}$ into $D$, i.e.

$$
h_{R}(r, \theta)=(\alpha+(\beta-\alpha)(r-R), \theta) .
$$

This map $h_{R}$ induces the map

$$
h_{R}^{*}: C_{0}^{1, \sigma}\left(\overline{D_{R}}\right) \rightarrow C_{0}^{1, \sigma}(\bar{D})
$$

defined by $h_{R}^{*}(u)(x)=u\left(h_{R}^{-1}(x)\right)$ for $x \in D$.

Then our equation (1.1) in $D_{R}$ becomes

$$
\begin{cases}L_{R} w=\lambda f(w) & \text { in } D, \\ w=0 & \text { on } \partial D\end{cases}
$$

where $w(x)=h_{R}^{*}(u)(x), L_{R}(w)=h_{R}^{*}\left(-\Delta\left(\left(h_{R}^{*}\right)^{-1}(w)\right)\right)$. Finally we can rewrite (4.1) as $w=T_{R}(w)$, where $T_{R}(w)=L_{R}^{-1}(\lambda f(w))$. It is easy to see that, for any $R>R_{*}, T_{R}$ is well defined $C_{0}^{1, \sigma}(\bar{D})$ into $C_{0}^{1, \sigma}(\bar{D})$ and is a compact operator. 
If $\mathcal{F}$ is an open bounded set in $C_{0}^{1, \sigma}(\bar{D})$ such that $I-T_{R} \neq 0$ on $\partial \mathcal{F}$, then the Leray-Schauder degree for the map $I-T_{R}$, i.e. $\operatorname{deg}\left(I-T_{R}, \mathcal{F}, 0\right)$ is well-defined.

Applying a result of [20] (see Proposition 2, p. 243) we have that

$$
\operatorname{deg}\left(I-T_{R}, \mathcal{F}, 0\right)=\operatorname{deg}\left(I-P_{R},\left(h_{R}^{*}\right)^{-1}(\mathcal{F}), 0\right)
$$

where $P_{R}(v)=(-\Delta)^{-1}(\lambda f(v))$ and it is defined in $C_{0}^{1, \sigma}\left(\overline{D_{R}}\right)$.

The operator $P_{R}$ is differentiable at $\bar{u}_{R}$, and as we have seen before the operator $I-P_{R}^{\prime}\left(\bar{u}_{R}\right)$ is invertible if $R \neq R_{k}$ and near $R_{k}, R_{k}>R_{*}$ is as in Corollary 3.3. Hence we have that, for any $R \neq R_{k}$ and near $R_{k}$,

$$
\operatorname{deg}\left(I-P_{R},\left(h_{R}^{*}\right)^{-1}(\mathcal{F}), 0\right)=\operatorname{deg}\left(I-P_{R}^{\prime}\left(\bar{u}_{R}\right),\left(h_{R}^{*}\right)^{-1}(\mathcal{F}), 0\right)=(-1)^{i(\lambda, \bar{u}}(44.3)
$$

if $\left(h_{R}^{*}\right)^{-1}(\mathcal{F})$ is a neighborhood of $\bar{u}_{R}$ in $C_{0}^{1, \sigma}\left(\overline{D_{R}}\right)$ such that $\bar{u}_{R}$ is the only solution of $\left(I-P_{R}\right)(u)=0$ in the closure of $\left(h_{R}^{*}\right)^{-1}(\mathcal{F})$.

Proof of Theorem 1.3 We consider the subspace $\mathcal{H}_{R}$ of $C^{1, \sigma}\left(\overline{D_{R}}\right)$ given by

$$
\begin{aligned}
\mathcal{H}_{R}: & =\left\{u \in C^{1, \sigma}\left(\overline{D_{R}}\right), u\left(x_{1}, \ldots, x_{N}\right)\right. \\
& \left.=u\left(g\left(x_{1}, \ldots, x_{N-1}\right), x_{N}\right) \text { for any } \in \in(N-1)\right\},
\end{aligned}
$$

where $O(N-1)$ is the orthogonal group in $\mathbb{R}^{N-1}$.

It follows from Proposition 5.2 of [26] that for any $i$ the eigenspace $V_{i}$ of the operator $-\Delta_{S^{N-1}}$, spanned by the eigenfunctions corresponding to the eigenvalue $\lambda_{i}$, which are $O(N-1)$ invariant, is one dimensional. Thus, the Morse index $i\left(\lambda, \bar{u}_{R}\right)$ in $\mathcal{H}_{R}$ grows by 1 when $R$ cross $R_{k}$. Then $i\left(\lambda, \bar{u}_{R_{k}+\epsilon}\right)=$ $i\left(\lambda, \bar{u}_{R_{k}-\epsilon}\right)+1$ if $\epsilon$ is small enough. Then for any positive, small enough $\epsilon$, letting $w_{R}=h_{R}^{*}\left(\bar{u}_{R}\right)$, we get from (4.2) and (4.3)

$$
\operatorname{deg}\left(I-T_{R_{k}-\epsilon}, \mathcal{F}_{R_{k}-\epsilon}, 0\right)=-\operatorname{deg}\left(I-T_{R_{k}+\epsilon}, \mathcal{F}_{R_{k}+\epsilon}, 0\right)
$$

if $\mathcal{F}_{R}$ is a neighborhood of $w_{R}$ in the space of functions which are $O(N-$ 1 ) invariant in $D$. This implies that there is a change in the degree at the point $\left(\lambda, R_{k}, w_{R_{k}}\right)$ and then bifurcation must occur. Moreover the bifurcating solutions are nonradial, since for each $R$ and $\lambda$ (1.1) admits exactly two radial solutions $\underline{u}_{R}$ and $\bar{u}_{R}$. This completes the proof.

\section{References}

[1] Bartsch, T., Clapp, M., Grossi, M., Pacella, F.: Asymptotically radial solutions in expanding annular domains. Math. Ann. 352, 485-515 (2012)

[2] Bandle, C., Coffman, C.V., Marcus, M.: Nonlinear elliptic problem in annular domains. J. Differ. Equ. 69, 332-345 (1987)

[3] Byeon, J.: Existence of many nonequivalent nonradial positive solutions of semilinear elliptic equations on three-dimensional annuli. J. Differ. Equ. 136, 136-165 (1997) 
[4] Crandall, M.G., Rabinowitz, P.H.: Some continuation and variational methods for positive solutions of nonlinear elliptic eigenvalue problems. Arch. Ration. Mech. Anal. 58, 207-218 (1975)

[5] Catrina, F., Wang, Z.Q.: Nonlinear elliptic equations on expanding symmetric domains. J. Differ. Equ. 156, 153-181 (1999)

[6] Dancer, E.N.: On non-radially symmetric bifurcation. J. Lond. Math. Soc. 20, 287-292 (1979)

[7] Dancer, E.N.: Some notes on the method of moving planes. Bull. Aust. Math. Soc. 46, 425-434 (1992)

[8] Du, Y.H., Dancer, E.N., Guo, Z.M.: Finite Morse index solutions of an ellptic equation with supercritical exponent. J. Differ. Equ. 250, 3281-3310 (2011)

[9] Du, Y.H., Guo, Z.M.: Finite Morse index solutions and asymptotics of weighted nonlinear elliptic equations. Adv. Differ. Equ. 18, 737-768 (2013)

[10] Du, Y.H., Guo, Z.M.: Finite Morse index solutions of weighted elliptic equations and the critical exponents. Calc. Var. PDEs 54, 3161-3181 (2015)

[11] Du, Y.H., Guo, Z.M., Wang, K.L.: Monotonicity formula and $\epsilon$-regularity of stable solutions to supercritical problems and applications to finite Morse index solutions. Calc. Var. PDEs 50, 615-638 (2014)

[12] Farina, A.: On the classification of solutions of Lane-Emden equation on unbounded domains of $\mathbb{R}^{N}$. J. Math. Pure. Appl. 87, 537-561 (2007)

[13] Garaizar, X.: Existence of positive radial solutions for semilinear elliptic equations in the annulus. J. Differ. Equ. 70, 69-92 (1987)

[14] Gladiali, F., Grossi, M., Pacella, F., Srikanth, P.N.: Bifurcation and symmetry breaking for a class of semilinear elliptic equations in an annulus. Calc. Var. PDEs 40, 295-317 (2011)

[15] Li, Y.Y.: Existence of many positive solutions of semilinear elliptic equations on annulus. J. Differ. Equ. 83, 348-376 (1990)

[16] Lin, S.S.: Positive radial solutions and non-radial bifurcation for semilinear elliptic equations in annular domains. J. Differ. Equ. 86, 367-391 (1990)

[17] Lin, S.S.: Existence of many positive nonradial solutions for nonlinear elliptic equations on an annulus. J. Differ. Equ. 103, 338-349 (1993)

[18] Lin, S.S.: Existence of positive nonradial solutions for nonlinear elliptic equations in annular domains. Trans. Am. Math. Soc. 332, 775-791 (1992)

[19] Lin, S.S.: Asymptotic behavior of positive solutions to semilinear elliptic equations on expanding annuli. J. Differ. Equ. 120, 255-288 (1995)

[20] Nussbaum, R.D.: The fixed point index for local condensing maps. Ann. Mat. Pura Appl. 89, 217-258 (1971) 
[21] Ni, W.M., Nussbaum, R.: Uniqueness and nonuniqueness for positive radial solutions of $\Delta u+f(u, r)=0$. Commun. Pure Appl. Math. 38, 67-108 (1985)

[22] Pacard, F.: Radial and non-radial solutions of $-\Delta u=\lambda f(u)$ on an annulus of $\mathbb{R}^{n}, n \geq 3$. J. Differ. Equ. 102, 103-138 (1993)

[23] Polacik, P., Quittner, P., Souplet, P.: Singularity and decay estimates in superlinear problems via Liouville-type theorems. Part I: Elliptic equations and systems. Duke Math. J. 139, 555-579 (2007)

[24] Rabinowitz, P.H.: Some global results for nonlinear eigenvalue problems. J. Funct. Anal. 7, 487-513 (1971)

[25] Rabinowitz, P.H.: Some aspects of nonlinear eigenvalue problems. Rock. Mount. J. Math. 3, 161-202 (1973)

[26] Smoller, J., Wasserman, A.: Symmetry-breaking for solutions of semilinear elliptic equations with general boundary conditions. Commun. Math. Phys. 105, 415-441 (1986)

Zongming Guo and Fangshu Wan

Department of Mathematics

Henan Normal University

Xinxiang 453007

People's Republic of China

e-mail: gzm@htu.cn

Fangshu Wan

e-mail: fangshuwan@163.com

Linfeng Mei

School of Mathematics \& Statistics

Qufu Normal University

Qufu 273165

People's Republic of China

e-mail: lfmei@outlook.com

Received: 9 May 2016.

Accepted: 12 October 2016. 